\title{
Antenatal Screening for Group B Streptococcus in the Setting of Preterm Premature Rupture of Membranes: Empiric versus Culture-based Prophylaxis
}

\author{
Leena B. Mithal, MD, MSCI ${ }^{1,2}$ Nirali Shah, MD $\quad$ Anna Romanova, MD ${ }^{1}$ Emily S. Miller, MD, MPH ${ }^{1,3}$ \\ ${ }^{1}$ Northwestern University Feinberg School of Medicine, Chicago, \\ Illinois \\ 2 Department of Pediatrics, Division of Infectious Diseases, Chicago, \\ Illinois \\ ${ }^{3}$ Department of Obstetrics and Gynecology, Division of Maternal- \\ Fetal Medicine, Chicago, Illinois \\ Address for correspondence Emily S. Miller, MD, MPH, 250 East \\ Superior Street, Suite 05-2146, Chicago IL 60611 \\ (e-mail: emily-miller-1@northwestern.edu).
}

Am J Perinatol Rep 2020;10:e26-e31.

\begin{abstract}
Keywords

- preterm premature rupture of membranes

- early onset neonatal sepsis

- group B streptococcusculture

Objective Imperfect culture sensitivity and increase of early onset neonatal sepsis (EONS) risk in preterm neonates raise concern that culture-based intrapartum antibiotic prophylaxis (IAP) may be insufficient after preterm premature rupture of membranes (PPROM). Our objective was to compare rates of EONS after empiric versus culture-based IAP in PPROM.

Study Design This retrospective cohort study included women with a singleton gestation and PPROM between 23 and 33 weeks. Outcomes after culture-based IAP were compared with empiric IAP. The primary outcome was EONS. Secondary outcomes included group B streptococcus (GBS) bacteremia, bacteremia, and neonatal GBS infection. Bivariable and multivariable logistic analyses were performed.

Results Of the 270 women who met inclusion criteria, 136 (50\%) had culture-based IAP of whom $36(26.5 \%)$ were GBS positive. There was no significant difference in bacteremia ( 2.2 vs. $4.5 \%, p=0.30$ ), GBS infection ( 0.8 vs. $0.7 \%, p=1.00$ ), or EONS (11.8 vs. $12.7 \%, p=0.82$ ) in infants of women with culture-based IAP compared with empiric IAP. Multivariable analysis confirmed a lack of advantage to empiric versus culture-based IAP in EONS risk (adjusted odds ratio $[\mathrm{aOR}]=0.82,95 \%$ confidence interval [CI]: 0.44-1.93).

Conclusion In pregnancies complicated by PPROM, infants of women who received culture-based IAP had no significant difference in EONS or GBS infection compared with infants of women with empiric IAP.
\end{abstract}

Group B streptococcus (GBS) bacteria colonizes the genital tract in 10 to $30 \%$ of pregnant women. ${ }^{1,2}$ GBS colonization during pregnancy is the primary risk factor for neonatal GBS infection with its accompanying risks of significant infant morbidity and mortality. ${ }^{3-5}$ Over the last several decades, intrapartum intravenous administration of antibiotics to women at risk for transmitting GBS has greatly reduced

received

December 19, 2018 accepted after revision October 15, 2019
DOI https://doi.org/

$10.1055 / \mathrm{s}-0039-3401807$. ISSN 2157-6998. neonatal disease. ${ }^{3,6,7}$ Current CDC guidelines recommend routine screening for GBS at 35 to 37 weeks of gestation and subsequent administration of intrapartum antibiotics if the GBS screen is positive (universal culture-based prophylaxis).

However, this approach is complicated in the setting of preterm premature rupture of membranes (PPROM) which causes up to $30 \%$ of preterm births. ${ }^{8}$ Prolonged rupture of
Copyright $\odot 2020$ by Thieme Medical Publishers, Inc., 333 Seventh Avenue, New York, NY 10001, USA. Tel: $+1(212) 760-0888$.
License terms

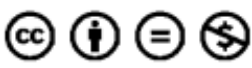


membranes ( $>12$ hours) is associated with increased risk of transmission of GBS to the newborn. ${ }^{9}$ Furthermore, preterm infants are at an increased risk of GBS infection, early-onset neonatal sepsis (EONS), and severe infection with complications, particularly at $<34$ weeks of gestational age (GA). 5,10,11

Women with PPROM will not yet typically have undergone routine outpatient GBS screening. In this case, the recommendation is to screen for GBS at the time of diagnosis of PPROM, prior to receipt of any antibiotics. Upon the diagnosis of labor or at the time of induction, the GBS status-if available-guides whether or not to use intravenous intrapartum antibiotics for GBS (i.e., a culture-based intrapartum antibiotic prophylaxis [IAP] approach). ${ }^{12}$ If culture results are not available, empiric IAP is provided. $3,13,14$

While screen-based IAP has been shown to be superior compared with the treatment based on risk factors, there are no adequate data regarding reliability of GBS screening in the setting of PPROM. ${ }^{15}$ Notably, in cases of neonatal GBS infections, up to 60 to $80 \%$ of women had a negative GBS screen. ${ }^{16}$ Thus, the limitations in sensitivity of GBS culture, particularly in the context of PPROM, may result in insufficient IAP for some women. ${ }^{7}$ Given the concerns for possible underdetection of GBS colonization and the increased morbidity and mortality associated with GBS infection in preterm neonates, it is currently unclear what the infectious morbidity is to neonates born to GBS-screen negative mothers. Therefore, the objective of this study was to evaluate if empiric IAP (administered universally to women with PPROM) is associated with reduced risk of EONS and neonatal GBS infection compared with culture-based IAP in the setting of PPROM.

\section{Methods}

\section{Study Design and Subjects}

We conducted a retrospective cohort study of women who delivered at Northwestern Memorial's Prentice Women's Hospital, a tertiary care hospital in Chicago, IL, between January 2010 and October 2015. Women were included if they were at least 18 years of age, carrying a singleton gestation, and were diagnosed with PPROM between 23 and 33 weeks. A total of 33 weeks was chosen as it afforded an opportunity for GBS cultures to result if they were sent. Mothers with suspected fetal anomalies were excluded.

Routine clinical care of women presenting with PPROM during the study period, in accordance with the American College of Obstetricians and Gynecologists (ACOG) guidelines, ${ }^{14}$ included clinical confirmation of the PPROM diagnosis and assessment of fetal well-being. If a GBS culture was obtained, it was done via rectal and vaginal swab prior to initiation of maternal antibiotics. The swab was incubated in LIM broth overnight and then subcultured to sheep blood agar plate. Colonies with $\beta$-hemolysis were identified with Vitek MS, an automated mass spectrometry microbial identification system. Antenatal betamethasone was administered and if labor had not ensued, latency antibiotics were initiated. These consisted of ampicillin and erythromycin for a total of 7 days. If PPROM was diagnosed prior to 32 weeks, women were started on magnesium for fetal neuroprotection and were monitored on the Labor and Delivery Unit for at least 12 hours. Thereafter, if maternal and fetal stability remained, they were transferred to the inpatient antepartum unit for close monitoring. Delivery was recommended if there was concern for chorioamnionitis (maternal temperature $\geq 100.4 \mathrm{~F}$ along with clinical signs and symptoms of intra-amniotic infection) or a clinically significant abruption. If preterm labor developed, women were transferred back to Labor and Delivery for monitoring but tocolysis was not administered. In the absence of a contraindication to expectant management or preterm labor, an induction was planned for 34 weeks of gestation. One rescue course of antenatal steroids was reserved for cases with the documentation of a clinical change which was suggestive of anticipated delivery within 7 days. Notably, during the study period, the decision to utilize empiric IAP or culture-based IAP in the setting of PPROM was left to the discretion of the attending obstetrician. According to Center for Diseases Control and Prevention guidelines in the United States, penicillin or ampicillin was used for prophylaxis in women without penicillin allergy. Alternate antibiotics include cefazolin in women at low risk of anaphylaxis, clindamycin, and vancomycin. ${ }^{3}$ All GBS cultures obtained and used for medical decision-making were obtained prior to initiation of any maternal antibiotics.

Comprehensive clinical data were abstracted from maternal and infant electronic medical records by authorized research personnel. Maternal data included sociodemographics, past medical history, past obstetrical history, labor, and delivery outcomes including length of antepartum admission, indication for delivery, development of fever or chorioamnionitis, and laboratory findings including GBS screening culture. Data on maternal antibiotic administration was abstracted, including GBS IAP and administration of latency antibiotics, for which standard of care was to administer ampicillin and erythromycin for 7 days. Neonatal data were obtained from linked chart within the electronic medical record system. Neonatal data included temperature abnormalities (recorded as the highest and the lowest temperature), clinical diagnosis of sepsis, any infectious workup results (e.g., CBC with differential, C-reactive protein [CRP], blood and cerebrospinal fluid cultures), and antibiotic and administration.

\section{Infant Outcomes}

The primary endpoint was EONS. Based on culture results, antibiotic treatment and laboratory data, patients were classified as having EONS using predetermined criteria based upon National Institute of Child Health and Human Development definitions for premature infants. ${ }^{11}$ EONS was defined as having received an antibiotic treatment course of $\geq 5$ days starting within 72 hours of life and either (1) a positive bacterial blood culture $\leq 72$ hours of life or (2) other abnormal laboratory results indicative of infection. Abnormal laboratory results indicative of infection were: $\mathrm{CRP} \geq 1 \mathrm{mg} / \mathrm{dL},{ }^{17}$ absolute neutrophil count outside normal range for $\mathrm{GA},{ }^{18,19}$ or immature-to-total (I:T) neutrophil ratio $>0.22 .{ }^{20}$ Analyses were done using one and two abnormal laboratory indices as criteria for presumed EONS. The secondary neonatal outcome measure was early onset neonatal GBS infection, defined as GBS bacteremia and/or meningitis. A subgroup analysis was conducted 
among women who were not on latency antibiotics at the time of delivery.

\section{Statistical Analyses}

Bivariate analysis of demographics, risk factors, and outcomes were conducted using Chi-squared or Fisher's exact tests for categorical variables and Student's t-test or Wilcoxon's rank sum tests for continuous variables. Multivariable analysis was performed to study the association between IAP strategy and EONS/GBS infection. $p$-values of $<0.05$ were considered statistically significant. STATA software, version 13.1 (College Station, TX) was used for this analysis.

\section{Ethics Statement}

The study was approved by the Northwestern University Feinberg School of Medicine Institutional Review Board prior to its initiation. Investigations were conducted according to the principles expressed in the Declaration of Helsinki.

\section{Results}

\section{Maternal Data}

There were 270 maternal/infant pairs that met inclusion criteria. Culture-based IAP was performed in 136 (50.4\%) women and the remaining 134 women were given empiric IAP. Of women with culture-based IAP strategy, 36 (26.5\%) were GBS positive and received antibiotics during labor and delivery. The remaining 100 did not receive IAP toward GBS prophylaxis. - Table 1 shows maternal characteristics. There

Table 1 Maternal Characteristics stratified by intrapartum antibiotic prophylaxis strategy

\begin{tabular}{|c|c|c|c|}
\hline & Empiric IAP & Culture-based IAP & \multirow[t]{2}{*}{$p$-Value } \\
\hline & $n=13$ & $n=136$ & \\
\hline $\begin{array}{l}\text { Advanced } \\
\text { maternal age }\end{array}$ & $25(18.7)$ & $25(18.4)$ & 0.95 \\
\hline Race/ethnicity & & & 0.85 \\
\hline $\begin{array}{l}\text { Non-Hispanic } \\
\text { white }\end{array}$ & $46(34.9)$ & $48(35.6)$ & \\
\hline $\begin{array}{l}\text { Non-Hispanic } \\
\text { black }\end{array}$ & $35(26.5)$ & $30(22.2)$ & \\
\hline Hispanic & $20(15.2)$ & $22(16.3)$ & \\
\hline Asian & $6(4.5)$ & $11(8.2)$ & \\
\hline $\begin{array}{l}\text { Other/ } \\
\text { unknown }\end{array}$ & $25(18.7)$ & $24(17.8)$ & \\
\hline Nulliparous & $76(56.7)$ & $80(58.8)$ & 0.73 \\
\hline $\begin{array}{l}\text { Prior preterm } \\
\text { birth }\end{array}$ & $28(20.9)$ & $24(17.7)$ & 0.50 \\
\hline $\begin{array}{l}\text { BMI at delivery } \\
\left(\mathrm{kg} / \mathrm{m}^{2}\right)\end{array}$ & $\begin{array}{l}29.0 \\
(25.1-32.6)\end{array}$ & $\begin{array}{l}28.5 \\
(25.2-32.9)\end{array}$ & 0.92 \\
\hline Tobacco use & $4(3.0)$ & $8(5.9)$ & 0.25 \\
\hline $\begin{array}{l}\text { Diabetes } \\
\text { (pregestational } \\
\text { or gestational) }\end{array}$ & $9(6.7)$ & $13(9.6)$ & 0.38 \\
\hline
\end{tabular}

Abbreviations: BMI, body mass index; IAP, intrapartum antibiotic prophylaxis. Note: data presented as $n$ (\%) or median (interquartile range).
Table 2 Antenatal and delivery data stratified by intrapartum antibiotic prophylaxis strategy

\begin{tabular}{|l|l|l|l|}
\hline \multirow{2}{*}{} & Empiric IAP & $\begin{array}{l}\text { Culture-based } \\
\text { IAP }\end{array}$ & \multirow{2}{*}{-Value } \\
\cline { 2 - 3 } & $\mathbf{n = 1 3 4}$ & $\boldsymbol{n}=\mathbf{1 3 6}$ & \\
\hline Antenatal steroids & $111(82.8)$ & $117(86.0)$ & 0.47 \\
\hline Induction of labor & $22(16.4)$ & $27(19.9)$ & 0.46 \\
\hline Preterm labor & $85(63.4)$ & $87(64.0)$ & 0.93 \\
\hline Cesarean delivery & $38(28.4)$ & $42(30.9)$ & 0.65 \\
\hline Chorioamnionitis & $18(13.4)$ & $21(15.4)$ & 0.64 \\
\hline $\begin{array}{l}\text { Gestational age } \\
\text { at delivery (wk) }\end{array}$ & $\begin{array}{l}32.0 \\
(29.9-33.1)\end{array}$ & $\begin{array}{l}31.9 \\
(29.3-33.5)\end{array}$ & 0.82 \\
\hline Infant sex (M) & $67(50.0)$ & $65(47.8)$ & 0.72 \\
\hline Birth weight & $1,773 \pm 515$ & $1,695 \pm 541$ & 0.23 \\
\hline
\end{tabular}

Abbreviation: IAP, intrapartum antibiotic prophylaxis.

Note: Data presented as $n(\%)$, median (interquartile range), or mean \pm standard deviation.

were no differences in advanced maternal age, race/ethnicity, parity, history of a prior preterm birth, body mass index at delivery, tobacco use, or diabetes between women who received culture-based IAP versus empiric IAP.

Details of the antenatal management and delivery were also largely similar between groups including receipt of a complete course of antenatal steroids, preterm labor, route of delivery, induction of labor, need for emergent delivery, and chorioamnionitis ( - Table 2). As anticipated, the duration of latency was longer in the culture-based IAP compared with the empiric IAP (7.6 vs. $2.1, p<0.001$ ). About $64 \%$ of women in the cohort were on latency antibiotics at the time of delivery and $87 \%$ of women in the cohort received antibiotics within 7 days of delivery; $72 \%$ in empiric IAP versus $57 \%$ in culture-based IAP group $(p=0.01)$. Infant sex, gestational age at delivery, and birth weight were similar between groups.

\section{Infant Outcomes}

Nine (3.3\%) infants in the cohort had bacteremia in the first 72 hours of life that was treated with an antibiotic course. The organisms isolated in blood culture from infants of mothers in the empiric IAP group $(n=6)$ were Escherichia coli $(n=3)$, GBS $(n=1)$, Klebsiella pneumoniae $(n=1)$, and coagulase negative staphylococcus species $(n=1)$. Bacteremia in the culturebased IAP group infants $(n=3)$ were $E$. coli $(n=1)$, GBS $(n=1)$, and Haemophilus influenzae $(n=1)$. There was no difference in the risk of early-onset GBS infection, and the risk of bacteremia between groups (6/134 vs. 3/136) was not statistically significant (adjusted odds ratio $[\mathrm{aOR}]=0.45,95 \%$ confidence interval [CI]: 0.11-1.87; - Table 3). Abnormal individual laboratory results indicative of infection (e.g., absolute neutrophil count, I:T ratio, and CRP) were also not significantly different between groups. We analyzed two grades of strictness of presumed EONS criteria by antibiotics and laboratory criteria. There was no increased risk of EONS by bacteremia and/or either one or two accessory laboratory criteria. While there were no differences identified in 
Table 3 Neonatal outcomes following empiric versus culture-based maternal intrapartum antibiotic prophylaxis

\begin{tabular}{|c|c|c|c|c|c|}
\hline & $\begin{array}{l}\text { Empiric IAP } \\
n=134(\%)\end{array}$ & $\begin{array}{l}\text { Culture-based IAP } \\
n=136(\%)\end{array}$ & $p$-Value & $\mathrm{aOR}^{\mathrm{a}}$ & $95 \% \mathrm{Cl}$ \\
\hline Neonatal bacteremia & $6(4.5)$ & $3(2.2)$ & 0.30 & 0.45 & $0.11-1.87$ \\
\hline GBS bacteremia & $1(0.7)$ & $1(0.8)$ & 1 & $\mathrm{~N} / \mathrm{A}$ & $\mathrm{N} / \mathrm{A}$ \\
\hline Antibiotics $\geq 5 \mathrm{~d}$ & $56(41.8)$ & $52(38.2)$ & 0.55 & 0.85 & $0.52-1.39$ \\
\hline Absolute neutropenia & $36(26.9)$ & $33(24.3)$ & 0.62 & 0.88 & $0.51-1.51$ \\
\hline Elevated I:T ratio & $33(24.6)$ & $32(23.5)$ & 0.83 & 0.91 & $0.51-1.62$ \\
\hline Abnormal C-reactive protein & $20(29.9)$ & $19(26.4)$ & 0.65 & 0.86 & $0.41-1.81$ \\
\hline EONS (culture and/or 2 abnormal laboratories) & $17(12.7)$ & $16(11.8)$ & 0.82 & 0.92 & $0.44-1.93$ \\
\hline EONS (culture and/or 1 abnormal laboratory) & $72(53.7)$ & $61(44.9)$ & 0.15 & 0.69 & $0.42-1.12$ \\
\hline
\end{tabular}

Abbreviation: aOR, adjusted odds ratio; $\mathrm{Cl}$, confidence interval; EONS, early onset neonatal sepsis; GBS, group B streptococcus; IAP, intrapartum antibiotic prophylaxis; I:T, immature-to-total ratio; N/A, not available.

${ }^{a}$ Adjusted for indication for delivery and gestational age at delivery.

bivariable analyses to inform potential confounders-given the strong empiric associates between the indication for delivery (i.e., preterm labor/chorioamnionitis vs. induction at 34 weeks) and gestational age at delivery and EONS-these variables were included as potential confounders in a multivariable regression. Our findings persisted after controlling for potential confounders ( - Table 3 ). A subgroup analysis, examining only women who were not still on latency antibiotics at the time of delivery, $(n=96)$ demonstrated no significant differences in EONS (defined by culture and/or one abnormal laboratory result) by IAP management strategy ( 47.4 vs. $46.6 \%$, $p=0.94$ ). Of note, the two cases of infant GBS infection were born to mothers on latency antibiotics at the time of delivery, one in each IAP group.

About $98 \%$ of infants had some systemic antibiotic exposure with 108 (40\%) having received an antibiotic course $\geq 5$ days. Early life antibiotic treatment of infants did not differ between groups, specifically infants receiving no antibiotics, $<5$ days of antibiotics (a "rule-out" of EONS), and $\geq 5$ days of antibiotics (an antibiotic treatment course for proven or presumed EONS), as shown in - Fig. 1. A post hoc power calculation demonstrated that this study had $90 \%$ power to detect at least a two-fold difference in EONS between the two IAP strategies.

\section{Discussion}

This study compares EONS and early-onset GBS infection in infants of mothers with PPROM prior to 33 weeks who received culture-based IAP versus those who received empiric IAP (without GBS screening culture sent). In our retrospective analysis, we found no significant difference in EONS or GBS infection between these IAP strategies. This study provides evidence supporting the current recommended guidelines for culture-targeted prophylaxis, even for women with PPROM and resultant preterm delivery.

The appropriate use of antibiotics in women with PPROM and high likelihood of preterm delivery is important for both maternal and infant outcomes. There are risks and benefits to widespread empiric antibiotic use, certainly administered directly to the infant, but also to mother prior to delivery. Risks include an increased risk of necrotizing enterocolitis in the

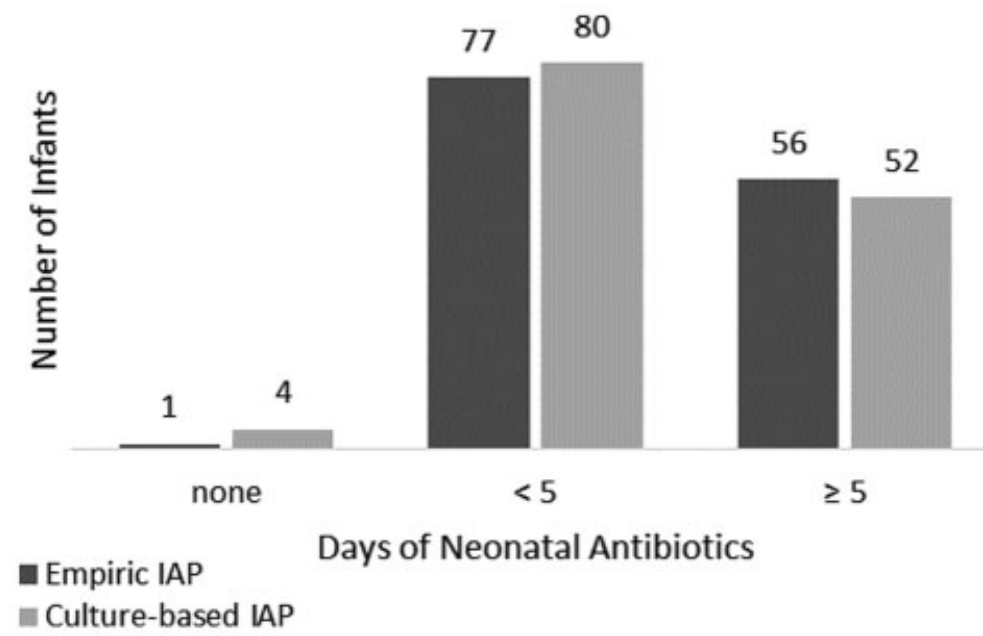

Fig. 1 Days of early life neonatal antibiotic administration. Number of infant antibiotic days, with course starting in first 72 hours of life (early life antibiotics). 
infant with early prolonged broad-spectrum antibiotics, ${ }^{21,22}$ and recent ongoing research suggests that maternal antibiotics prior to delivery are associated with notable alternations in an infant's early life microbiome diversity. These microbial community changes have potential far-reaching effects on an infant's metabolic, immune, and allergic health. ${ }^{23}$ Administration of certain antibiotics, however, have been shown to prolong gestational latency, important in reducing morbidities of extreme prematurity. ${ }^{14,24,25}$ There has also been a significant decrease in GBS disease since institution of GBS IAP recommendations. Thus, antibiotic treatment in appropriate circumstances improves neonatal outcomes.

To date, there has been a paucity of data on the optimal strategy for IAP administration for women with PPROM, who do not have GBS culture results available at the time of rupture. The theoretical possibility of an increased risk of neonatal infection compounded by the imperfect sensitivity of culture particularly in the setting of PPROM led to this retrospective study. The negative predictive value of GBS culture declines outside a 5-week window between culture and delivery; thus, early screening would not address this problem. Our study reinforces that screening culture and culture result-based IAP decisions are not causing a missed opportunity for EONS prevention. Although our study was retrospective and culture proven, EONS is relatively infrequent; the point estimates of the incidence of bacteremia cases were lower in the culture-based IAP compared with empiric IAP cases. This finding provides reassurance that culture-based IAP is unlikely to result in an increased risk of EONS. Although our demographics demonstrate no difference in preterm versus induction of labor, it is possible that there was a shorter latency period and more rapid spontaneous preterm labor in the empiric IAP group-perhaps a reflection of underlying pathology related to increase risk of bacteremia. Recognizing the imperfect sensitivity of blood culture for EONS, we included presumed EONS based on laboratory criteria in our analyses and found no difference between IAP strategies. Furthermore, GBS infection was not different between groups which are the primary objective of the CDC's IAP guidelines.

The majority of women in both groups received antibiotics to promote gestational latency which remains standard clinical practice. There were significantly more women in the empiric IAP group who received latency antibiotics in the week preceding delivery. How latency antibiotics $>7$ days from delivery versus active treatment with latency antibiotics in the peripartum period effects EONS and neonatal GBS infection is unknown. Latency antibiotics could play a confounding or interaction role in the association between IAP and EONS, and thus requires further study.

The strengths of this study include detailed maternal and infant data on demographics, risk factors, antibiotics, and infant outcomes for a cohort of maternal-infant dyads. The granular definitions of EONS, incorporating both proven and presumed sepsis, microbiology results, and clinical antibiotic treatment decision strengthen the analysis of neonatal outcomes. A notable limitation in this study is the few infants with proven early-onset GBS infection and culture-proven infection in general, which limits our power to detect potentially clinically important differences in this relatively rare but highly morbid condition.

In conclusion, this study demonstrates that a screen and culture-based IAP strategy for women presenting with PPROM at $<33$ weeks appears to be appropriate, without obvious missed opportunities for neonatal infection prevention and equivalent overall outcomes. Further data on timing of culture-based screening and other antibiotic treatments for PPROM management in larger prospective observational studies are necessary to advance perinatal care and optimize targeted antibiotic exposures to mother and infant.

\section{Funding}

Eunice Kennedy Shriver National Institute of Child Health and Human Development (grant/award number: “K12HD050121-09”), and National Institutes of Health's National Center for Advancing Translational Sciences (grant/award number: "UL1TR001422”).

\section{Conflict of Interest \\ None declared.}

\section{References}

1 Regan JA, Klebanoff MA, Nugent RP; Vaginal Infections and Prematurity Study Group. The epidemiology of group B streptococcal colonization in pregnancy. Obstet Gynecol 1991;77(04):604-610

2 Campbell JR, Hillier SL, Krohn MA, Ferrieri P, Zaleznik DF, Baker CJ. Group B streptococcal colonization and serotype-specific immunity in pregnant women at delivery. Obstet Gynecol 2000;96(04):498-503

3 Verani JR, McGee L, Schrag SJ; Division of Bacterial Diseases, National Center for Immunization and Respiratory Diseases, Centers for Disease Control and Prevention (CDC). Prevention of perinatal group B streptococcal disease-revised guidelines from CDC, 2010. MMWR Recomm Rep 2010;59(RR-10):1-36

4 Schuchat A. Epidemiology of group B streptococcal disease in the United States: shifting paradigms. Clin Microbiol Rev 1998;11 (03):497-513

5 Phares CR, Lynfield R, Farley MM, et al; Active Bacterial Core surveillance/Emerging Infections Program Network. Epidemiology of invasive group B streptococcal disease in the United States, 1999-2005. JAMA 2008;299(17):2056-2065

6 Lim DV, Morales WJ, Walsh AF, Kazanis D. Reduction of morbidity and mortality rates for neonatal group B streptococcal disease through early diagnosis and chemoprophylaxis. J Clin Microbiol 1986;23(03):489-492

7 Matorras R, García-Perea A, Omeñaca F, Diez-Enciso M, Madero R, Usandizaga JA. Intrapartum chemoprophylaxis of early-onset group B streptococcal disease. Eur J Obstet Gynecol Reprod Biol 1991;40(01):57-62

8 Goldenberg RL, Culhane JF, lams JD, Romero R. Epidemiology and causes of preterm birth. Lancet 2008;371(9606):75-84

9 Adair CE, Kowalsky L, Quon H, et al. Risk factors for early-onset group B streptococcal disease in neonates: a population-based case-control study. CMAJ 2003;169(03):198-203

10 Schrag SJ, Farley MM, Petit S, et al. Epidemiology of invasive earlyonset neonatal sepsis, 2005 to 2014. Pediatrics 2016;138(06): e20162013

11 Stoll BJ, Hansen NI, Sánchez PJ, et al; Eunice Kennedy Shriver National Institute of Child Health and Human Development Neonatal Research Network. Early onset neonatal sepsis: the burden of group B Streptococcal and E. coli disease continues. Pediatrics 2011;127(05):817-826 
12 Cagno CK, Pettit JM, Weiss BD. Prevention of perinatal group B streptococcal disease: updated CDC guideline. Am Fam Physician 2012;86(01):59-65

13 American College of Obstetricians and Gynecologists Committee on Obstetric Practice. ACOG committee opinion no. 485: prevention of early-onset group B streptococcal disease in newborns. Obstet Gynecol 2011;117(04):1019-1027

14 Bulletins ACoOaGCoP. Practice bulletin no. 172: premature rupture of membranes. Obstet Gynecol 2017;128(04):e165-e177

15 Schrag SJ, Zell ER, Lynfield R, et al; Active Bacterial Core Surveillance Team. A population-based comparison of strategies to prevent early-onset group B streptococcal disease in neonates. N Engl J Med 2002;347(04):233-239

16 Van Dyke MK, Phares CR, Lynfield R, et al. Evaluation of universal antenatal screening for group B streptococcus. N Engl J Med 2009; 360(25):2626-2636

17 Benitz WE, Han MY, Madan A, Ramachandra P. Serial serum Creactive protein levels in the diagnosis of neonatal infection. Pediatrics 1998;102(04):E41

18 Manroe BL, Weinberg AG, Rosenfeld CR, Browne R. The neonatal blood count in health and disease. I. Reference values for neutrophilic cells. J Pediatr 1979;95(01):89-98

19 Hornik CP, Benjamin DK, Becker KC, et al. Use of the complete blood cell count in early-onset neonatal sepsis. Pediatr Infect Dis J 2012;31(08):799-802
20 Schelonka RL, Yoder BA, Hall RB, et al. Differentiation of segmented and band neutrophils during the early newborn period. J Pediatr 1995;127(02):298-300

21 Owen J, Groome LJ, Hauth JC. Randomized trial of prophylactic antibiotic therapy after preterm amnion rupture. Am J Obstet Gynecol 1993;169(04):976-981

22 Kenyon SL, Taylor DJ, Tarnow-Mordi W, Group OC; ORACLE Collaborative Group. Broad-spectrum antibiotics for preterm, prelabour rupture of fetal membranes: the ORACLE I randomised trial. Lancet 2001;357(9261):979-988

23 Rock RB-AH, Neuman H, Hanya H, Koren O, Youngster I. The impact of antibiotic use in breastfeeding women on the infant's gut microbiome:a prospective cohort study. Open Forum Infect Dis 2017;4:232

24 Lorthe E, Ancel PY, Torchin H, et al. Impact of latency duration on the prognosis of preterm infants after preterm premature rupture of membranes at 24 to 32 weeks' gestation: a national populationbased cohort study. J Pediatr 2017;182:47-52.e2

25 Mercer BM, Miodovnik M, Thurnau GR, et al; National Institute of Child Health and Human Development Maternal-Fetal Medicine Units Network. Antibiotic therapy for reduction of infant morbidity after preterm premature rupture of the membranes. A randomized controlled trial. JAMA 1997;278(12): 989-995 